\title{
Therapy for Late Stage Melanoma
}

\section{James L Cox}

Department of Biochemistry, AT Still University, Kirksville, Missouri, USA

"Corresponding author: James L Cox, Department of Biochemistry, AT Still University, Kirksville, Missouri, USA, Tel: 6606262466; E-mail: jcox@atsu.edu Received date: August 19, 2015; Accepted date: October 17, 2015, 2015; Published date: October 24, 2015

Copyright: $\odot 2015$ Cox JL. This is an open-access article distributed under the terms of the Creative Commons Attribution License, which permits unrestricted use, distribution, and reproduction in any medium, provided the original author and source are credited.

Keywords: Melanoma; Immunotherapy

\section{Introduction}

Melanoma arises from mutated melanocytes which have progressed to cancer. Late stage (stage III-IV) melanoma is typically not curable with surgery because of its spread. It is one of the most highly invasive cancers and is also extremely resistant to cancer cell killing with chemotherapeutic agents. Metastatic melanoma is a deadly disease for which there are no effective therapies. The situation of therapy has improved, however, recently in that there is a fraction of long-term survivors with remission in patients undergoing immunotherapy [1]. Also, targeted therapy is gaining attention as a new option for melanoma therapy, especially when problems with resistance, which often occurs, can be overcome. Here, a brief overview of melanoma therapy will include a discussion of new directions for therapy on the horizon, including some of our current work in this area.

Some major therapies for late stage melanoma are covered here, including some recent experimental therapies:

\section{Dacarbazine}

For four decades dacarbazine has been used for single agent chemotherapy for melanoma [2]. It is a methylating agent which targets DNA and inhibits DNA replication thus blocking melanoma cell proliferation. No other chemotherapeutic agent, when given as a single drug, has proven superior to dacarbazine. Single-agent dacarbazine at $1000 \mathrm{mg} / \mathrm{m}^{2}$ has been suggested as reference standard treatment for metastatic melanoma [3]. Therapeutic combinations which include dacarbazine have not proven to be superior to its use as a single agent, especially considering toxic side effects seen with certain combinations of agents [4].

High-dose IL-2 IL-2 activates cytotoxic T cells and NK cells and has been in use since FDA approval in 1998. Long-term remissions have been observed in a small subset of melanoma patients with IL-2 treatment [5]. Side effects of IL-2 monotherapy include vomiting, nausea, and fatigue, thus limiting dosing regimens. Similar outcomes for interferon $\alpha$ have been observed in clinical studies [6].

\section{Vemurafenib}

The 2002 finding that over 50\% of melanomas are BRAF V600E mutants ushered in a new era in targeted therapy [7]. Vemurafenib was introduced as a BRAF V600E antagonist which is active in many melanoma patients. Side effects of the drug include skin reactions, photosensitivity, and athralgia. A major drawback to venurafenib use is the rapid development of resistance (months) in most patients [8]. Most resistance stems from activation of some component of the MAPK pathway or other signaling pathway. Attempts to overcome resistance by adding another agent, such as a MEK inhibitor, have proven somewhat successful, but work is on-going [9]. Resistance to targeted therapy can be either intrinsic or acquired. Next generation sequencing of melanoma patient samples should greatly augment our ability to predict effective therapeutic agents which overcome resistance to targeted therapy.

\section{Ipilimumab}

Introduction of effective immunotherapy in 2011 with ipilimumab ushered in a new phase for melanoma therapy. Previous attempts at immunization against melanoma had not proven all that successful. Ipilimumab, a monoclonal antibody against CTLA-4, changed the target to an immune checkpoint in the activation of $\mathrm{T}$ cells. In phase III clinical trials $11 \%$ of patients had long term remissions and for the first time overall survival increased for metastatic melanoma patients [10]. Side effects of ipilimumab are stomach pain with diarrhea or constipation. Rarely, there may be fatal outcomes due to excessive Tcell activation. At the present time Ipilimumab and several other new checkpoint inhibitors are the most promising therapies for metastatic melanoma. New combinatorial therapies that include checkpoint inhibitors are currently being tested $[11,12]$.

\section{Apoptosis induction}

Resistance to apoptosis or programmed cell death is a key feature of melanoma. The goal of almost all melanoma therapy is to induce apoptosis in the metastatic disease and hence eliminate it without any undue cytotoxic effects. A vital question is how can apoptosis be induced selectively in melanoma cells, particularly since melanoma exhibits intrinsic resistance to the process? One way forward is to find agents which block melanoma survival signaling such as through MAPK and PI3K signaling pathways. Work is currently in the preclinical stage for the development of new agents that will block survival signaling for melanoma $[13,14]$.

\section{Oncolytic viruses}

A number of different viruses are being tested as lytic, cell killing agents for a wide variety of cancers, including melanoma. Cancers lose their resistance to virus replication during cancer progression. A major advantage of the use of viruses is that they can be used as expression vectors also for a wide variety of therapeutic genes. Efforts have been made to create therapeutic viruses that will not only destroy melanoma cancer cells but also induce an immune response to the tumor for systemic control of the disease. The oncolytic virus furthest along (phase III clinical testing) is known as T-vec [15]. It is a herpes simplex virus that expresses an inserted gene for GM-CSF. There is great potential in this line of attack as the therapeutic efficacy has just begun to be realized. 


\section{Vaccines for melanoma}

Numerous attempts have been made to create a vaccine for melanoma with disappointing and mixed responses the usual outcome [16]. The tumor microenvironment is such an immune suppressive area that even if an effective immune response to a tumor could be produced it would be largely blunted. Some attempts to boost tumor immunity by administering the immunomodulatory GM-CSF or interferon a have produced systemic responses. Sipuleucel-T is a dendritic cell-based vaccine that has demonstrated improved survival for prostate cancer [17]. Efforts are underway to test sipuleucel-T for melanoma. Application of monoclonal antibodies directed to tumor antigens has had minor impact for melanoma [18]. Solid tumors in general are less responsive than hematological malignancies to monoclonal antibody based therapies. Peptides and proteins used for vaccination alone often yield a weak immune response [19]. A large number of adjuvants have been used to increase immune responses for cancer vaccines. Several toll-like receptor ligands are being used as adjuvants with pleiotrophic effects [20]. Again, it is the tumor-induced immunosuppression which limits the effects of T-cell mediated tumor rejection.

\section{Metastasis inhibitors}

Genes which encode inhibitors of metastasis are known but have not yet been introduced to clinical use because our knowledge of just what these inhibitors are and their mechanisms of action is small. Ideally inhibitors of metastasis would block tumor growth at secondary sites of spread with very little toxicity. This might be achieved by induction of apoptosis by blocking growth factor signaling in some manner. Agents are also critically needed that could kill dormant metastatic cells since most chemotherapeutic agents work primarily for dividing cells. Metastasis suppressor genes are a group of over 20 genes that are defined by their ability to inhibit metastasis without affecting primary tumor growth [21]. While advances have been made for determining actions of the various metastasis suppressor genes, we are some distance from clinical application of knowledge for melanoma therapy. It is clear the mechanisms of action for the metastasis suppressor genes are complex in that they may control multiple cellular pathways [22]. Some type of gene therapy, or better, gene reactivation of dormant metastasis suppressor genes might be of tremendous benefit for melanoma therapy. Perhaps the information gained on metastasis suppressor gene action will also provide new targets for interfering with metastasis of melanoma.

\section{Angiogenesis inhibitors}

Angiogenesis is the formation of new blood vessels to supply nutrients to a growing tumor. Melanoma is a highly angiogenic cancer type. The goal, however, of eliminating or even controlling melanoma through anti-angiogenic agents seems to be fraught with difficulties. First, there is a plethora of endogenous factors which stimulate angiogenesis such that inhibiting one factor may be insufficient to totally arrest angiogenesis [23]. Second, melanoma can develop resistance to anti-angiogenic agents making long term treatment problematical [24]. Third, melanoma may be able to co-opt preexisting blood vessels making anti-angiogenesis strategies less effective [25]. Currently, clinical trials incorporate an anti-angiogenic agent such as bevicezumab in with several other chemotherapeutic agents to help shrink growth of the melanoma. Perhaps as anti-angiogenic strategies continue to improve we will see better control over melanoma tumor growth in clinical trials.

\section{Inhibition of melanoma metastasis- cystatin C}

Work from our laboratory first showed the small, cysteine protease inhibitor, cystatin $\mathrm{C}$, is able to inhibit the metastasis of B16 melanoma [26]. In the inhibition of metastasis, cystatin primarily inhibited melanoma cell migration and increased apoptosis of the metastatic melanoma cells in vivo [27]. One conclusion from our work was that it did not appear cystatin $\mathrm{C}$ was inhibiting metastasis by acting as a cysteine protease inhibitor. Although cysteine proteases are often overexpressed by many cancers, their contributions to metastasis seemed inconsistent with the potent inhibitory effects of cystatin [27]. More recent work by our laboratory has demonstrated the active segment of cystatin $\mathrm{C}$ is the conserved QVVAG region and not sequences required for protease inhibition (in preparation). It will be of great interest to find the mechanism for anti-metastatic actions of cystatin. In mice, overexpression of cystatin $\mathrm{C}$ in liver with viral expression vector infection was able to inhibit metastasis of breast cancer [28]. Delivery of cystatin to a cancer patient, it is hoped, may someday be able to arrest the spread of melanoma and contribute to effective therapy for this deadly disease.

\section{Conclusion}

The advent of immunotherapy with ipilimumab and related strategies has ushered in a new era of treatment for metastatic melanoma. New targeted therapies, such as that for BRAF, will come about due to an increased understanding of the molecular basis of melanoma. Currently, new combinations of agents with immunotherapy may be even more promising to move forward towards a cure for what was until recently an incurable disease.

\section{References}

1. Madorsky Rowdo FP, Baron A, Urrutia M, Mordoh J (2015) Immunotherapy in Cancer: A Combat between Tumors and the Immune System; You Win Some, You Lose Some. Front Immunol 6: 127.

2. Tawbi HA, Kirkwood JM (2007) Management of metastatic melanoma. Semin Oncol 34: 532-545.

3. Chapman PB, Einhorn LH, Meyers ML, Saxman S, Destro AN, et al. (1999) Phase III multicenter randomized trial of the Dartmouth regimen versus dacarbazine in patients with metastatic melanoma. J clin oncol 17: 2745-2751.

4. Daponte A, Signoriello S, Maiorino L, Massidda B, Simeone E, et al. (2013) Phase III randomized study of fotemustine and dacarbazine versus dacarbazine with or without interferon- $\alpha$ in advanced malignant melanoma. J Transl Med 11: 38.

5. Atkins MB, Lotze MT, Dutcher JP, Fisher RI, Weiss G, et al. (1999) Highdose recombinant interleukin 2 therapy for patients with metastatic melanoma: analysis of 270 patients treated between 1985 and 1993. J Clin Oncol 17: 2105-2116.

6. Tarhini AA, Gogas H, Kirkwood JM (2012) IFN- $\alpha$ in the treatment of melanoma. J Immunol 189: 3789-3793.

7. Davies H, Bignell GR, Cox C, Stephens P, Edkins S, et al. (2002) Mutations of the BRAF gene in human cancer. Nature 417: 949-954.

8. Hauschild A, Grob JJ, Demidov LV, Jouary T, Gutzmer R, et al. (2012) Dabrafenib in BRAF-mutated metastatic melanoma: a multicentre, openlabel, phase 3 randomised controlled trial. Lancet 380: 358-365.

9. Bhatia S, Tykodi SS, Lee SM, Thompson JA (2015) Systemic therapy of metastatic melanoma: on the road to cure. Oncology (Williston Park) 29: 126-135.

10. Hodi FS, O'Day SJ, McDermott DF, Weber RW, Sosman JA, et al. (2010) Improved survival with ipilimumab in patients with metastatic melanoma. N Engl J Med 363: 711-723. 
11. Larkin J, Hodi FS, Wolchok JD (2015) Combined Nivolumab and Ipilimumab or Monotherapy in Untreated Melanoma. N Engl J Med 373: 1270-1271.

12. Maio M, Grob JJ, Aamdal S, Bondarenko I, Robert C, et al. (2015) Fiveyear survival rates for treatment-naive patients with advanced melanoma who received ipilimumab plus dacarbazine in a phase III trial. J Clini Oncol 33: 1191-1196.

13. Mohana-Kumaran N, Hill DS, Allen JD, Haass NK (2014) Targeting the intrinsic apoptosis pathway as a strategy for melanoma therapy. Pigment Cell Melanoma Res 27: 525-539.

14. Mukherjee N, Reuland SN, Lu Y, Luo Y, Lambert K, et al. (2015) Combining a BCL2 inhibitor with the retinoid derivative fenretinide targets melanoma cells including melanoma initiating cells. J Invest Dermatol 135: 842-850.

15. Andtbacka RH, Kaufman HL, Collichio F, Amatruda T, Senzer N, et al. (2015) Talimogene Laherparepvec Improves Durable Response Rate in Patients With Advanced Melanoma. J Clin Oncol 33: 3377.

16. Ott PA, Fritsch EF, Wu CJ, Dranoff G (2014) Vaccines and melanoma. Hematol Oncol Clin North Am 28: 559-569.

17. Tse BW, Jovanovic L, Nelson CC, de Souza P, Power CA, et al. (2014) From bench to bedside: immunotherapy for prostate cancer. Biomed Res Int 2014: 981434.

18. Jilaveanu LB, Aziz SA, Kluger HM (2009) Chemotherapy and biologic therapies for melanoma: do they work? Clin Dermatol 27: 614-625.

19. Alexandrescu DT, Ichim TE, Riordan NH, Marincola FM, Di Nardo A, et al. (2010) Immunotherapy for melanoma: current status and perspectives. J Immunother 33: 570-590.
20. Adams S, O'Neill DW, Nonaka D, Hardin E, Chiriboga L, et al. (2008) Immunization of malignant melanoma patients with full-length NYESO-1 protein using TLR7 agonist imiquimod as vaccine adjuvant. J immunol 181: 776-784.

21. Stafford LJ, Vaidya KS, Welch DR (2008) Metastasis suppressors genes in cancer. Int J Biochem Cell Biol 40: 874-891.

22. Hurst DR, Welch DR (2011) Metastasis suppressor genes at the interface between the environment and tumor cell growth. Int Rev Cell Mol Biol 286: 107-180.

23. Tomanek RJ, Schatteman GC (2000) Angiogenesis: new insights and therapeutic potential. Anat Rec 261: 126-135.

24. van Beijnum JR, Nowak-Sliwinska P1, Huijbers EJ1, Thijssen VL1, Griffioen AW2 (2015) The great escape; the hallmarks of resistance to antiangiogenic therapy. Pharmacol Rev 67: 441-461.

25. Ziyad S, Iruela-Arispe ML (2011) Molecular mechanisms of tumor angiogenesis. Genes Cancer 2: 1085-1096.

26. Cox JL, Sexton PS, Green TJ, Darmani NA (1999) Inhibition of B16 melanoma metastasis by overexpression of the cysteine proteinase inhibitor cystatin C. Melanoma Res 9: 369-374.

27. Ervin H, Cox JL (2005) Late stage inhibition of hematogenous melanoma metastasis by cystatin C over-expression. Cancer Cell Int 5: 14.

28. Kopitz C, Anton M, Gansbacher B, Krüger A (2005) Reduction of experimental human fibrosarcoma lung metastasis in mice by adenovirus-mediated cystatin $\mathrm{C}$ overexpression in the host. Cancer Res 65: 8608-8612. 